\title{
Prefrailty and Chronic Morbidity in the Youngest Old: An Insight from the Lausanne Cohort Lc65+
}

\author{
Nadia Danon-Hersch, MD, MSc, * Nicolas Rodondi, MD, MAS, ${ }^{\dagger}$ Jacques Spagnoli, MSc, * and \\ Brigitte Santos-Eggimann, MD, DrPH, MPH*
}

OBJECTIVES: To estimate the prevalence of prefrailty, frailty, comorbidity, and disability in the youngest old and to identify chronic diseases associated with individual frailty criteria.

DESIGN: Population-based cohort study of noninstitutionalized elderly adults at baseline; cross-sectional analysis.

SETTING: Lausanne, Switzerland.

PARTICIPANTS: One thousand two hundred eighty-three individuals with complete data on frailty, aged 65 to 70 (58.5\% women).

MEASUREMENTS: Frailty was assessed according to an adaptation of Fried's criteria (shrinking, weakness, exhaustion, slowness, and low activity, three criteria needed for the diagnosis of frailty, 1 to 2 for prefrailty). Other outcomes were diseases diagnosed by a doctor $(\geq 2$ chronic diseases: comorbidity) and limitations in activities of daily living (ADLs, basic and instrumental).

RESULTS: At baseline, of 1,283 participants $71.1 \%$ were classified as nonfrail, $26.4 \%$ as prefrail, and $2.5 \%$ as frail. The proportion of women increased across these three groups $(56.5 \%, 62.8 \%$, and $71.9 \%$, respectively; $P=.01)$, as did the proportion of individuals with one or more chronic diseases $(68.0 \%, 82.8 \%$, and $90.6 \%$, respectively; $P<.001)$ and the proportion with basic or instrumental ADL disability $(1.6 \%, 10.3 \%$, and $59.4 \%$, respectively; $P<.001$ ). Weakness (low grip strength) was the most frequent criterion $(14.3 \%)$. Prefrail participants had significantly more comorbidity and ADL disability than nonfrail participants $(P<.001)$. When present in isolation, weakness was associated with two to three times greater prevalence of coronary heart disease, other heart diseases, diabetes mellitus, and arthritis. Similarly, a significant association was identified between exhaustion and depression.

From the "Institute of Social and Preventive Medicine, University of Lausanne Hospital Center, and 'Department of Ambulatory Care and Community Medicine, University of Lausanne, Lausanne, Switzerland.

Address correspondence to Nadia Danon-Hersch, Institute of Social and Preventive Medicine (IUMSP), University of Lausanne Hospital Centre, Route de la Corniche 10, 1010 Lausanne, Switzerland. E-mail: Nadia. Danon@chuv.ch

DOI: $10.1111 /$ j.1532-5415.2012.04113.x
CONCLUSION: Prefrailty is common in the youngest old. The most prevalent frailty criterion is weakness, which is associated with cardiovascular diseases. Longitudinal studies of the evolution of prefrailty should explore the role of potential interactions between individual frailty criteria and specific chronic diseases. J Am Geriatr Soc 60:1687-1694, 2012.

Key words: prefrailty; frailty; disability; chronic disease; youngest old; Switzerland

$T$ he concept of frailty has been developed in geriatrics during past decades ${ }^{1}$ to define a state of vulnerability and loss of adaptation to stress. ${ }^{2-4}$ Different models ${ }^{1}$ have been used to explain the development of frailty. Fried and colleagues, considering frailty as a biological syndrome, suggested the occurrence of a pathophysiological "cycle of frailty associated with declining energetics and reserve" ${ }^{2}$ and proposed an operational definition for a frailty phenotype that relies on five criteria: slowness, weakness, weight loss, low level of physical activity, and self-reported exhaustion. ${ }^{2}$ Individuals with at least three of the five criteria are classified as frail, and those fulfilling one or two criteria are considered prefrail or intermediate. The frailty phenotype independently predicts recurrent falls, ${ }^{5}$ disability, ${ }^{2,6,7}$ hip fracture, ${ }^{5,6}$ hospitalization, ${ }^{2}$ nursing home admission, ${ }^{7}$ and death. ${ }^{2,5-9}$

Nevertheless, many gaps remain in the understanding of the physiopathology of frailty. Although the risk of adverse outcomes seems to increase gradually from the state of nonfrailty to prefrailty and frailty, ${ }^{2}$ little is known about the significance of prefrailty. This intermediate stage may provide insights into the mechanisms involved. The stage of prefrailty also deserves special interest because frailty is a continuous process that may be partly reversible, especially in its initial phases. ${ }^{10}$

Whereas the association between cardiovascular disease and frailty has been demonstrated, ${ }^{11,12}$ the current 
study aimed to identify which frailty criteria were more specifically linked with cardiovascular disease. Likewise, although the association between other chronic diseases (e.g., arthritis, diabetes mellitus, chronic obstructive pulmonary disease) and frailty has been established, ${ }^{2,13-15}$ it was hypothesized that specific chronic diseases might be more tightly linked with one or another frailty criterion. These associations might be involved in different parts of the frailty cycle and could provide indications regarding the physiopathology of frailty.

In this report, it was hypothesized that prefrailty would not be rare in the community-dwelling population aged 65 to 70 ; the prevalence of chronic diseases and disability would differ significantly between prefrail and nonfrail populations; and the distribution of chronic diseases and disability would differ between individuals with different frailty criteria, even among prefrail individuals experiencing a single frailty criterion. To test these hypotheses, the data from the population-based Lausanne cohort Lc65+ were analyzed. ${ }^{16}$

\section{METHODS}

\section{Design}

The Lausanne cohort Lc65+ is a longitudinal, observational study started in 2004 at the University of Lausanne Hospital Center (Switzerland). ${ }^{16}$ It aims to investigate the determinants, manifestations, and outcomes of frailty from its earliest stage in the general population. A sample of 1,564 individuals representative of the general community-dwelling population has been enrolled and is currently being followed from age 65 to death. The ethics committee of the Faculty of Biology and Medicine of the University of Lausanne has approved the study protocol.

\section{Recruitment Process and Inclusion Criteria}

Individuals were enrolled at the age of 65 to 70 and provided written informed consent to participate. ${ }^{16}$ To be included in 2004, participants had to be residents of Lausanne (city of 125,000 inhabitants) born between 1934 and 1938. Exclusion criteria at enrollment were being institutionalized or unable to respond because of advanced dementia. Enrollment has been previously described. ${ }^{16}$ Of the 3,056 people who were initially mailed questionnaires, $2,096(69 \%)$ replied, of whom $1,564(75 \%)$ agreed to participate. ${ }^{16}$ Overall, nonparticipants had demographic characteristics similar to those of participants; ${ }^{16}$ only $8 \%$ of those refusing to participate attributed their refusal to poor health, ${ }^{16}$ and $58 \%$ had "a general reluctance to participate in any survey." Of the 1,564 respondents to the initial questionnaire, $1,524(97.4 \%)$ were still eligible, ${ }^{16}$ and 1,422 $(93.3 \%)$ participated in the baseline assessment in 2005 .

The present analysis, which focused on prefrailty, included all participants with complete data for frailty classification (nonfrail, prefrail, or frail; $\mathrm{n}=1,283$ ).

\section{Assessment Process}

Baseline data were collected in 2004 using a self-administered questionnaire sent to participants' homes, followed by an interview at the study center with measurements and per- formance tests conducted by trained medical assistants in 2005. The questionnaire included items used in the Swiss health surveys, Monitoring of Trends and Determinants in Cardiovascular Disease ${ }^{17}$ and the Survey of Health, Aging and Retirement in Europe (SHARE). ${ }^{18}$ Items on selfreported medical diagnoses of diseases ("Has a doctor ever told you that you had...?") assessed coronary heart disease (CHD), other heart diseases, stroke, diabetes mellitus, hypertension, chronic respiratory disease, osteoporosis, arthritis, cancer, gastrointestinal ulcer, depression, and hypercholesterolemia. In accordance with the lists of medical diagnoses used in SHARE ${ }^{19,20}$ and the Cardiovascular Health Study (CHS), ${ }^{2}$ only eight of these diagnoses were included in the variable "number of chronic diseases": $\mathrm{CHD}$, other heart diseases, stroke, diabetes mellitus, hypertension, chronic respiratory disease, arthritis, and cancer.

Disability in basic or instrumental activities of daily living (ADLs or IADLs) was assessed according to two questions: "During the last four weeks, did you have difficulty with performing the following activities: taking a shower or a bath, getting dressed, eating, getting in/out of bed or an arm-chair, using the toilets?" (ADLs), and "During the last four weeks, did you have difficulty shopping or performing your usual tasks at home?" (IADLs). There were three possible answers: No, I have had no difficulty at all; I have had difficulties with one or more of these activities, but I didn't get help; and I have received help with one or more of these activities. Participants who had received help were considered to have disability.

\section{Frailty Assessment}

The clinical assessment was conducted following a standardized protocol. ${ }^{16}$ Frailty was assessed at baseline according to the five dimensions of the phenotype described by Fried, $^{2}$ although these dimensions were operationalized with partly different criteria; Table 1 , reproduced from a previous publication about Lc65+, ${ }^{16}$ details how frailty characteristics were measured in $\mathrm{CHS}^{2}$ and in Lc65+. Participants with three to five frailty criteria in Lc65+ were categorized as frail, and those with one or two criteria were categorized as prefrail.

\section{Statistical Analysis}

Statistical analyses were performed using Stata 12 software (Stata Corp, College Station, TX). Results were expressed as absolute numbers and percentages. Bivariate comparisons were performed using the chi-square test or Fisher exact test for categorical variables. Multivariate odds ratios (ORs) and 95\% confidence intervals (CIs) of the prevalence of self-reported chronic diseases were calculated in participants with zero or one frailty criterion using multivariate logistic regression analysis adjusted for sex and all five frailty criteria.

\section{RESULTS}

\section{Characteristics of the Sample}

The study sample initially included all participants who participated at baseline in the Lc65+ cohort in 2004/05 
Table 1. Operationalization of Frailty Characteristics in the Cardiovascular Health Study (CHS) ${ }^{2}$ and the Lausanne cohort Lc65+ Study, Reproduced from Santos-Eggimann et al. ${ }^{16}$

\begin{tabular}{|c|c|c|}
\hline \multirow[b]{2}{*}{ Frailty } & \multicolumn{2}{|c|}{ Criteria } \\
\hline & Cardiovascular Health Study & Lausanne Cohort Lc65+ Study \\
\hline Shrinking & Unintentional weight loss $>10$ pounds in prior year & Any reported unintentional weight loss in prior year \\
\hline Weakness & $\begin{array}{l}\text { Grip strength: lowest } 20 \% \text { (according to sex and body mass } \\
\text { index) }\end{array}$ & $\begin{array}{l}\text { Grip strength: application of CHS sex- and body mass index- } \\
\text { specific cutoff values }^{\mathrm{a}}\end{array}$ \\
\hline Slowness & Walking time/15 feet: slowest 20\% (by gender, height) & $\begin{array}{l}\text { Walking time/20 m: application of CHS gender- and height- } \\
\text { specific cutoff values }\end{array}$ \\
\hline Low activity & $\begin{array}{l}\text { Physical activity self-report: lowest } 20 \% \mathrm{kcal} / \text { week expenditure, } \\
\text { according to sex, estimated from the short version of the } \\
\text { Minnesota Leisure Time Activity questionnaire }\end{array}$ & $\begin{array}{l}\text { Physical activity self-report: (i) doing }<20 \text { minutes of sports } \\
\text { per week, (ii) walking }<90 \text { minutes per week, and (iii) } \\
\text { avoidance of climbing stairs or carrying light loads in daily } \\
\text { activities }^{b}\end{array}$ \\
\hline $\begin{array}{l}\text { Intermediate, } \\
\text { possibly } \\
\text { prefrail }\end{array}$ & $1-2$ criteria present & $1-2$ criteria present \\
\hline Frail & 3-5 criteria present & 3-5 criteria present \\
\hline
\end{tabular}

${ }^{a}$ The grip strength test was performed on the right hand ${ }^{46}$ (using the best of three measurements).

b Low activity was defined when all three statements were fulfilled. This measurement of physical activity based on three questions has been adapted from the Monitoring of Trends and Determinants in Cardiovascular Disease Physical Activity Questionnaire ${ }^{17,47}$ to suit activity patterns of individuals aged $65-70$.

and were evaluated for frailty $(\mathrm{n}=1,283)$. Birth years were evenly distributed throughout the 1934-1938 period, and $58.5 \%$ of participants were women, closely reflecting proportions observed in this age range in the community-dwelling population of Lausanne. Of the 1,283 participants, $71.1 \%$ were classified as nonfrail, $26.4 \%$ as prefrail, and $2.5 \%$ as frail (Table 2). No participant had all five criteria of the frailty phenotype. Weakness was the most frequent criterion and was experienced by $14.3 \%$ of Lc65+ participants $(\mathrm{n}=183)$, followed by weight loss $(\mathrm{n}=115,9.0 \%)$, low activity $(\mathrm{n}=88,6.9 \%)$, exhaustion $(\mathrm{n}=81,6.3 \%)$, and slowness $(\mathrm{n}=43,3.4 \%)$. One-third $(34.9 \%)$ reported two or more chronic conditions diagnosed by a physician, and $37.5 \%$ reported a single chronic condition. Hypertension, hypercholesterolemia, and arthritis were most often reported. Most participants were independent in their daily life; $3.7 \%$ had received help during the four previous weeks for IADLs only and an additional $1.6 \%$ for ADLs.

Most prefrail participants also had at least one chronic disease; $27.5 \%$ of all participants had none of the eight listed chronic diseases: $32.0 \%$ of the nonfrail, $17.2 \%$ of the prefrail, and $9.4 \%$ of the frail (test for trend $P<.001$, Table 2). Of the 1,282 participants with complete data regarding the three health dimensions, 288 $(22.5 \%)$ had no frailty criterion, no disability, and no chronic disease. Of the 338 prefrail participants with complete data, $57(16.9 \%)$ had no disability and no chronic disease.

\section{Characteristics of Prefrail Individuals}

Prefrail participants differed significantly from nonfrail participants in many respects (Table 2). Prefrail participants were more frequently female and were older. Fewer than $2 \%$ of nonfrail participants needed help in IADLs $(1.4 \%)$ and ADLs $(0.2 \%)$, more than $10 \%$ of prefrail participants did so (IADL, 7.4\%; ADLs, $2.9 \% ; P<.001$ ). Prefrail individuals also reported comorbidity $(\geq 2$ chronic diseases) more often. Specific health conditions that were more frequently reported in prefrail participants than in nonfrail participants included CHD $(P=.04)$, other heart diseases $(P<.001)$, stroke $(P=.01)$, diabetes mellitus $(P<.001)$, hypertension $(P<.001)$, chronic respiratory diseases $(P=.02), \quad$ osteoporosis $(P=.01), \quad$ arthritis $(P<.001)$, and depression $(P<.001)$.

The proportion of women increased across the three frailty categories (nonfrail, 56.5\%; prefrail, 62.8\%; frail, $71.9 \%$; test for trend $P=.01$ ), as did the proportion of older participants (born in 1934: nonfrail, $16.9 \%$; prefrail, $23.9 \%$; frail, $28.1 \%$; test for trend $P<.001$ ).

Across frailty categories, there were increasing trends in the prevalence of disability (test for trend $P<.001$ ), comorbidity (test for trend $P<.001$ ), CHD (test for trend $P<.001$ ), other heart diseases (test for trend $P<.001$ ), stroke (test for trend $P<.001$ ), diabetes mellitus (test for trend $P<.001$ ), hypertension (test for trend $P<.001$ ), chronic respiratory disease (test for trend $P=.001$ ), osteoporosis (test for trend $P=.006$ ), arthritis (test for trend $P<.001$ ), and depression (test for trend $P<.001$; Table 2 ). 
Table 2. Demographic and Health Characteristics at Baseline (2004/5) in Nonfrail, Prefrail (1 or 2 Frailty Criteria), and Frail (3-5 Criteria) Participants

\begin{tabular}{|c|c|c|c|c|c|c|}
\hline Characteristic & $\begin{array}{c}\text { Nonfrail, } \\
\mathrm{n}=912 \mathrm{n}(\%)\end{array}$ & $\begin{array}{l}\text { Prefrail, } \\
\mathrm{n}=\mathbf{3 3 9}\end{array}$ & $\begin{array}{l}\text { Frail, } \\
\mathrm{n}=32\end{array}$ & $\begin{array}{c}\text { Total, } \\
\mathbf{N}=\mathbf{1 , 2 8 3}\end{array}$ & $\begin{array}{l}\chi^{2} \text { P-Value (Nonfrail } \\
\text { vs Prefrail) }\end{array}$ & $\begin{array}{c}\text { Test for Trend } P \text {-value (Nonfrail, } \\
\text { Prefrail, and Frail) }\end{array}$ \\
\hline Female & $515(56.5)$ & $213(62.8)$ & $23(71.9)$ & $751(58.5)$ & .04 & .01 \\
\hline \multicolumn{7}{|l|}{ Birth year } \\
\hline 1938 & $197(21.6)$ & $52(15.3)$ & $3(9.4)$ & $252(19.6)$ & .001 & $<.001$ \\
\hline 1937 & $194(21.3)$ & $51(15.0)$ & $3(9.4)$ & $248(19.3)$ & & \\
\hline 1936 & $178(19.5)$ & 76 (22.4) & $12(37.5)$ & $266(20.7)$ & & \\
\hline 1935 & $189(20.7)$ & 79 (23.3) & $5(15.6)$ & $273(21.3)$ & & \\
\hline 1934 & $154(16.9)$ & $81(23.9)$ & $9(28.1)$ & $244(19.0)$ & & \\
\hline \multicolumn{7}{|c|}{ Help received with ADLs or IADLs } \\
\hline None & $897(98.4)$ & $304(89.7)$ & $13(40.6)$ & $1,214(94.6)$ & $<.001^{\mathrm{a}}$ & $<.001$ \\
\hline With IADLs only & $13(1.4)$ & $25(7.4)$ & $10(31.3)$ & $48(3.7)$ & & \\
\hline With ADLs & $2(0.2)$ & $10(2.9)$ & $9(28.1)$ & $21(1.6)$ & & \\
\hline \multicolumn{7}{|c|}{ Number of chronic diseases ${ }^{b}$} \\
\hline 0 & $292(32.0)$ & $58(17.2)$ & $3(9.4)$ & $353(27.5)$ & $<.001$ & $<.001$ \\
\hline 1 & $359(39.4)$ & $118(34.9)$ & $4(12.5)$ & $481(37.5)$ & & \\
\hline$\geq 2$ & $261(28.6)$ & $162(47.9)$ & $25(78.1)$ & $448(34.9)$ & & \\
\hline \multicolumn{7}{|c|}{ Self-reported medical diagnoses } \\
\hline $\begin{array}{l}\text { Coronary heart } \\
\text { disease }\end{array}$ & $72(7.9)$ & $39(11.5)$ & 10 (31.3) & $121(9.4)$ & .04 & $<.001$ \\
\hline $\begin{array}{l}\text { Other heart } \\
\text { diseases }^{c}\end{array}$ & $45(4.9)$ & 41 (12.1) & $5(15.6)$ & $91(7.1)$ & $<.001$ & $<.001$ \\
\hline Stroke & $11(1.2)$ & $11(3.3)$ & $9(28.1)$ & $31(2.4)$ & .01 & $<.001$ \\
\hline Diabetes mellitus & $71(7.8)$ & $52(15.4)$ & $6(18.8)$ & $129(10.1)$ & $<.001$ & $<.001$ \\
\hline Hypertension & $334(36.6)$ & $162(47.9)$ & $20(62.5)$ & $516(40.3)$ & $<.001$ & $<.001$ \\
\hline Hypercholesterolemia & $320(35.1)$ & $127(37.6)$ & $14(43.8)$ & $461(36.0)$ & .41 & .24 \\
\hline $\begin{array}{l}\text { Chronic respiratory } \\
\text { disease }\end{array}$ & $62(6.8)$ & $37(10.9)$ & $6(18.8)$ & $105(8.2)$ & .02 & .001 \\
\hline Osteoporosis & $90(9.9)$ & $50(14.8)$ & $6(18.8)$ & $146(11.4)$ & .01 & .006 \\
\hline Arthritis & 291 (31.9) & $154(45.6)$ & $17(53.1)$ & $462(36.0)$ & $<.001$ & $<.001$ \\
\hline Cancer & 104 (11.4) & 45 (13.3) & 7 (21.9) & $156(12.2)$ & .35 & .09 \\
\hline Gastrointestinal ulcer & $51(5.6)$ & $25(7.4)$ & $4(12.5)$ & $80(6.2)$ & .24 & .08 \\
\hline Depression & $101(11.1)$ & $63(18.6)$ & $7(21.9)$ & 171 (13.3) & $<.001$ & $<.001$ \\
\hline
\end{tabular}

The chi-square $\left(\chi^{2}\right)$ test compares proportions in the group of nonfrail participants with the group of prefrail participants.

${ }^{\text {a }}$ Fisher exact two-tailed test (if expected frequency $<5$ in any of the cells).

${ }^{b}$ All chronic diseases were self-reported medical diagnoses. The number of chronic diseases included coronary heart disease, other heart diseases, stroke, diabetes mellitus, hypertension, chronic respiratory disease, arthritis, and cancer (maximum number $=8$ ).

${ }^{\mathrm{c}}$ Congestive heart failure, cardiac valvular disease, and heart muscle disease.

$\mathrm{ADL}=$ activity of daily living; $\mathrm{IADL}=$ instrumental activity of daily living.

\section{Characteristics of Prefrail Individuals Fulfilling a Single Criterion}

When considering the 252 participants with a single frailty criterion, $102(40.5 \%)$ had weakness, $73(29.0 \%)$ had weight loss, $43(17.1 \%)$ had low activity, $29(11.5 \%)$ had exhaustion, and five $(2.0 \%)$ had slowness. The 102 persons with weakness as the sole frailty criterion differed significantly from nonfrail participants: $66.7 \%$ were women (vs $56.5 \%$ of nonfrail participants, chi-square $P=.048$ ), and they were older than nonfrail participants $(P=.01)$. Individuals experiencing weakness alone needed help with ADLs and IADLs more frequently $(6 \%$ for IADLs only, $4 \%$ for ADLs, $P<.001$, Table 3$)$. They more frequently reported two or more chronic diseases $(P<.001)$. Weakness was significantly associated with $\mathrm{CHD}(P=.003)$, other heart diseases $(P=.001)$, diabetes mellitus $(P=.04)$, and arthritis $(P=.001)$. Multivariate logistic regression analysis (adjusted for sex and all five frailty criteria, results not shown in Table 3, but available upon request) indicated that low grip strength was associated with two to three times greater prevalence of CHD (multivariate $\mathrm{OR}=2.9,95 \% \mathrm{CI}=1.6-5.3$ ), other heart diseases (multivariate $\mathrm{OR}=2.9,95 \% \mathrm{CI}=1.5-5.6$ ), diabetes mellitus (multivariate $\mathrm{OR}=2.2,95 \% \mathrm{CI}=1.2-4.1)$, and arthritis (multivariate $\mathrm{OR}=1.9,95 \% \quad \mathrm{CI}=1.2-2.8$ ). The 29 participants with exhaustion as the single frailty criterion reported depression $(P=.01)$ and receiving help with ADLs and IADLs $(P=.04)$ significantly more often than nonfrail participants (multivariate $\mathrm{OR}$ for the prevalence of depression $=3.0,95 \% \mathrm{CI}=1.3-7.0)$.

\section{DISCUSSION}

In this cross-sectional analysis of the Lc65+ cohort study at baseline, $26 \%$ of all participants were prefrail, and $2 \%$ were frail. Prefrail individuals had significantly more comorbidity and ADL and IADL disability than nonfrail participants. Weakness was the most frequent frailty criterion, and when present in isolation, it was associated with two to three times greater prevalence of $\mathrm{CHD}$, other heart diseases, diabetes mellitus, and arthritis. Similarly, a 
Table 3. Comparison of the Prevalence of Chronic Diseases and Disability in Nonfrail Participants $(\mathbf{n}=912)$ and in Participants with a Single Frailty Criterion $(\mathrm{n}=252)$

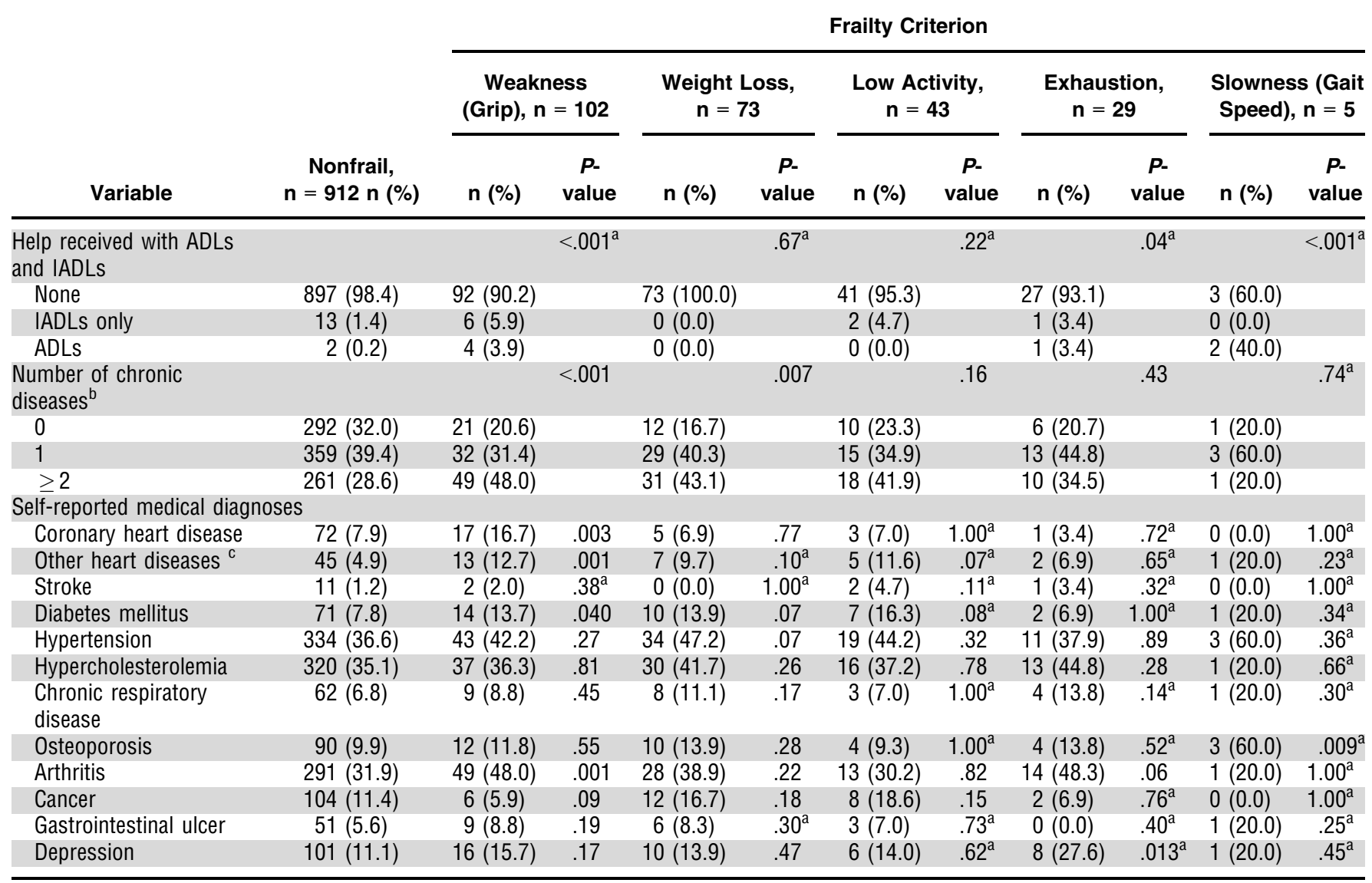

$P$-values are from the chi-square test for comparison of proportions with nonfrail participants.

${ }^{a}$ Fisher exact two-tailed test (if expected frequency $<5$ in any of the cells).

${ }^{b}$ All chronic diseases are self-reported medical diagnoses. The number of chronic diseases included coronary heart disease, other heart diseases, stroke, diabetes mellitus, hypertension, chronic respiratory disease, arthritis, and cancer (maximum number $=8$ ).

${ }^{\mathrm{c}}$ Congestive heart failure, cardiac valvular disease, and heart muscle disease.

$\mathrm{ADL}=$ activity of daily living; $\mathrm{IADL}=$ instrumental activity of daily living.

significant association was identified between exhaustion and depression. The existence of associations between individual frailty criteria and specific chronic diseases suggests that they could result from common pathophysiological changes. Further exploration of these processes in longitudinal studies may contribute to understanding the natural history of frailty.

\section{Prevalence of Frailty, Disability, and Comorbidity at Baseline}

In this "young old" age group (65-70), frailty was uncommon $(2 \%)$, whereas one-quarter were prefrail. Any attempt to compare prevalence estimates across studies should be conducted with caution, because the definitions of the frailty criteria, the distribution of confounders (e.g., age and sex), and exclusion criteria differ between studies. Prefrailty prevalence in Lc65+ appeared to be lower than in other studies, which reported a prevalence ranging from $43 \%$ to $54 \% .^{2,5,21-23}$ In SHARE, prefrailty prevalence after age 65 ranged from $35 \%$ in Germany to $51 \%$ in Spain, with a north-south gradient, ${ }^{18}$ but these studies included older individuals (mean age from $74^{18,21}$ to $82^{5,22,23}$ ). In the estimation of the authors of the current study, the prevalence of prefrailty among CHS participants aged 65 to 74 should have approximated $44 \% .^{2}$ This sample included older individuals than in Lc65+, as well as a minority cohort of 687 African American men and women (who have higher frailty prevalence), and the frailty criteria measured in $\mathrm{CHS}^{2}$ and Lc65+ were different.

In Lc65+, $48 \%$ of prefrail and $78 \%$ of frail individuals reported at least two chronic diseases; corresponding percentages in CHS were $54 \%$ and $68 \%$, ${ }^{2}$ although CHS had a somewhat different list of chronic diseases (validated by clinical tests ${ }^{2}$ ) and other exclusion criteria (Parkinson's disease; Mini-Mental State Examination score less than 18; use of carbidopa-levodopa, donepezil, or antidepressants). In a Taiwanese health survey of elderly adults, ${ }^{15} 76 \%$ of prefrail and $85 \%$ of frail individuals had two or more selfreported chronic diseases, although the definition of chronic disease was broader, including osteoporosis and cataracts. Disability prevalence (basic or instrumental) appeared to be lower in Lc65+ prefrail participants $(10 \%)$ than in CHS $(29 \%),{ }^{2}$ and the prevalence of instrumental 
disability in Lc65+ $(7 \%)$ seemed to be lower than in the Osteoporotic Fractures in Men Study ${ }^{8}$ (MrOS, 20\%) or the MOBILIZE Boston Study ${ }^{24}(28 \%)$, although disability was defined more strictly in Lc65+ (having received help with ADLs in the past 4 weeks) than in $\mathrm{CHS}^{2}$ or MOBILIZE $^{24}$ (difficulty in $\geq 1$ ADLs). CHS $^{2}$ and MOBILIZE ${ }^{24}$ also included older participants. Increasing trends in the prevalence of comorbidity and disability across frailty categories confirm previous results. ${ }^{2,15}$

\section{The Most Frequent Frailty Criterion in Prefrail Participants}

In Lc65+, the most frequent frailty criterion (alone and in association with other criteria) was weakness, followed by shrinking, low activity, exhaustion, and slowness. Weakness prevalence appeared lower in Lc65+ $(14 \%)$ than in the 10 countries of SHARE (26\%) but similar to Switzerland alone $(17 \%) .{ }^{18}$ Despite the younger age of Lc65+ participants, weakness prevalence seemed slightly higher in Lc65+ participants than in the men of $\operatorname{MrOS}(13 \%),{ }^{8}$ possibly because weakness is more frequent in women ${ }^{25}$ (a finding also observed in Lc65+). Shrinking seemed slightly more prevalent in Lc65+ (any unintentional weight loss over the past year, $9 \%$ ) than in MOBILIZE $(7 \%)^{24}$ or the Hertfordshire Cohort Study $(4-5 \%),{ }^{25}$ but these studies defined shrinking as a weight loss of more than 10 pounds during the past year. The prevalence of exhaustion (determined with slightly different sentences) appeared to be similar in Lc65+ $(6 \%)$, MOBILIZE $(9 \%),{ }^{24}$ the Hertfordshire Cohort Study (6\% in men, $10 \%$ in women), ${ }^{25}$ and MrOS $(8 \%){ }^{8}$ Slowness was present in $3 \%$ of Lc65+ participants, $3 \%$ of MrOS participants, ${ }^{8}$ and $8 \%$ of the participants in a German study on prefrail individuals ${ }^{26}$ that included institutionalized participants and had differing exclusion criteria.

Although several articles have identified the most frequent criterion in specific age groups, ${ }^{2,8,18,24,26}$ to the knowledge of the authors of the current study, not many studies have identified the first frailty criterion. In the Women's Health and Aging Study II (WHAS II), ${ }^{27}$ a longitudinal study with a 7.5-year follow-up period that included 420 nonfrail women aged 70 to 79 at baseline, weakness was the most common initial manifestation. Although weakness had the highest prevalence in Lc65+, the crosssectional design of the present report precludes the conclusion that it is the first criterion to appear. This hypothesis must be evaluated in the follow-up data. Grip strength has already been identified as a powerful independent predictor of disability ${ }^{28}$ and of cause-specific and total mortality. ${ }^{29}$

\section{The Chronic Diseases Most Frequently Associated with Prefrailty in Lc65+}

Prefrail Lc65+ participants had a significantly higher prevalence of $\mathrm{CHD}$, other heart diseases, stroke, diabetes mellitus, hypertension, chronic respiratory disease, osteoporosis, arthritis, and depression than nonfrail individuals, findings also observed in $\mathrm{CHS}^{2}$ and WHAS II. $^{27}$ As in $\mathrm{CHS}^{2}$ and WHAS $\mathrm{II}^{27}$ prefrail participants had a significantly higher prevalence of disability than nonfrail participants.
Previous studies have reported associations between chronic diseases ${ }^{2,12,26,27,30}$ and prefrailty but not with individual frailty criteria. In Lc65+, low grip strength was associated with a significantly greater prevalence of CHD, other heart diseases, diabetes mellitus, and arthritis. The link between frailty and mortality has often been described as being mediated through the cardiovascular system (subclinical disease). ${ }^{12}$ Of the pathways suggested to explain this association, atherosclerosis, a state of chronic inflammation resulting in a loss of lean mass (among other consequences), has been proposed. ${ }^{12}$ The association between low grip strength and cardiovascular diseases observed in Lc65+ is consistent with this hypothesis. In addition, the association between diabetes mellitus and low grip strength has long been reported, ${ }^{31}$ and individuals with diabetes mellitus develop the conditions necessary for frailty earlier than other individuals as they age. ${ }^{13}$

Exhaustion as a single frailty criterion was significantly associated with depression, an association previously observed. ${ }^{26}$ A Taiwanese survey also reported a high prevalence of depressive symptoms in frail $(89 \%)$ and prefrail $(32 \%)$ elderly adults. ${ }^{15}$ In Lc65+, $19 \%$ of prefrail participants reported depression (vs $11 \%$ of nonfrail participants, $P<.001)$. Although the cause of its association with frailty remains unknown, depression has a recognized prognostic value. $^{32}$

These cross-sectional associations between individual frailty criteria and distinct chronic diseases deserve additional study in longitudinal analyses. Arthritis may be a local and straightforward explanation for weakness, and this frailty criterion may not be specific. The independent prognostic significance of weakness as a sole frailty criterion should be evaluated before weakness alone can be considered a target for prevention.

\section{Limitations}

Of all 3,056 individuals contacted, 1,422 (47\%) completed the baseline assessment. This final participation rate was comparable with other surveys involving communitydwelling individuals (rather than hospitalized persons) in Western countries; ${ }^{33-35}$ for example, in CHS, ${ }^{33} 31 \%$ of those contacted in the randomly selected sample were enrolled. Studies looking for systematic differences in health status between participants and nonparticipants yielded inconsistent results. ${ }^{34,36-38}$ The nonparticipation rate seems to be higher in lower socioeconomic groups. ${ }^{38}$ There might have been a selection bias due to differential participation rates across frailty categories in Lc65+, although only $8 \%$ of those refusing to participate attributed their refusal to poor health. ${ }^{16}$ The prevalence of frailty, comorbidity, and disability may have been underestimated if affected individuals had lower participation rates than healthy persons, which could affect the generalizability of the findings.

Although Lc65+ frailty criteria differed partly from the criteria that Fried and colleagues proposed, ${ }^{2}$ different operationalizations of the phenotype's dimensions reflect the same underlying mechanisms (e.g., weight loss, sarcopenia, and diminution of the reserve capacity). The consistency of observations across diverse studies with various measurement instruments supports the plausibility of the 
findings. These limitations (e.g., potential selection bias and different definitions of frailty criteria) might have affected prevalence estimates for prefrailty but are unlikely to explain the existence of associations between individual frailty criteria and specific chronic diseases. Weakness was involved in most of the associations observed in Lc65+, and it was measured using Fried's exact definition.

Assessment of comorbidity was based on self-reporting of medical diagnoses. Self-reported diseases are often used in population-based studies ${ }^{15,19,20}$ and seem to be accurate $^{39-41}$ for specific diagnoses. Studies have observed substantial ${ }^{41-43}$ agreement between self-reporting and medical reporting of medical conditions, particularly for lifethreatening, acute-onset diseases (e.g., myocardial infarction and stroke) and chronic symptomatic disorders requiring ongoing management (e.g., diabetes mellitus). ${ }^{39,44,45}$ Participants usually underreport hypertension, ${ }^{45}$ and agreement on heart failure differs between studies. ${ }^{40,43}$ When compared with general practitioners' information, the accuracy of patient self-reporting is substantial for cancer but poor for arthritis. ${ }^{39}$ Nonetheless, errors in disease measurement due to self-reporting are unlikely to have a differential distribution across frailty categories; underreporting of some conditions should have led to nondifferential misclassification, attenuating the strength of associations between isolated frailty criteria and specific diseases.

\section{Strengths}

Unlike other studies, ${ }^{2,21-23}$ this study population had a narrow age range (65-70), which limits the potential for age-related confounders or biases, such as selection bias due to differential participation rates across age groups. ${ }^{33}$

Except for institutionalization and advanced dementia, this study did not exclude individuals with specific diseases, whereas other studies excluded persons with terminal cancer, ${ }^{15}$ arthritis, angina pectoris, chronic obstructive pulmonary disease, or depression ${ }^{26}$ or those taking antidepressants. $^{2}$ The design of the current study allowed associations between individual frailty criteria and chronic diseases to be examined. Furthermore, the limited number of exclusion criteria led to a study sample that was more representative of the source population.

\section{CONCLUSION}

Frailty is rare in the youngest old, whereas prefrailty is common. Weakness is the most frequent frailty criterion; when present in isolation, it is significantly associated with CHD, other heart diseases, diabetes mellitus, and arthritis. Likewise, exhaustion is associated with depression. Longitudinal studies regarding the evolution of prefrail persons should explore the role of potential interactions between individual frailty criteria and specific chronic diseases.

\section{ACKNOWLEDGMENTS}

The authors thank all Lc65+ participants for their involvement in the study. We are grateful to the medical assistants who conducted the tests at the study center and to those who assisted us in data acquisition.
Conflict of Interest: The editor in chief has reviewed the conflict of interest checklist provided by the authors and has determined that the authors have no financial or any other kind of personal conflicts with this paper.

The data used in this manuscript stem from the Lc65+ cohort study, a project exclusively funded by public institutions. Since 2004, the Lc65+ project has been funded by the Swiss National Foundation for Scientific Research (3247B0-120795/1); the Faculty of Biology and Medicine, University of Lausanne; the Loterie Romande (a nonprofit organization supporting research and social projects); the Fondation Médecine Sociale et Préventive, Lausanne; University of Lausanne Hospital Centre and its Institute of Social and Preventive Medicine; the Service of Geriatric Medicine and Geriatric Rehabilitation; University of Lausanne Department of Ambulatory Care and Community Medicine; Canton de Vaud Department of Public Health; and the City of Lausanne.

Author Contributions: Study design: Santos-Eggimann. Data acquisition: Santos-Eggimann and Spagnoli. Data analysis and interpretation: Santos-Eggimann, DanonHersch, Spagnoli, and Rodondi. Manuscript preparation: Danon-Hersch, Santos-Eggimann, Spagnoli, and Rodondi.

Sponsor's Role: The sponsors played no role in the study design, methods, data analysis, or manuscript preparation.

\section{REFERENCES}

1. Hogan DB, MacKnight C, Bergman H. Models, definitions, and criteria of frailty. Aging Clin Exp Res 2003;15:1-29.

2. Fried LP, Tangen CM, Walston J et al. Frailty in older adults: Evidence for a phenotype. J Gerontol A Biol Sci Med Sci 2001;56A:M146-M156.

3. Fried LP, Ferrucci L, Darer J et al. Untangling the concepts of disability, frailty, and comorbidity: Implications for improved targeting and care. J Gerontol A Biol Sci Med Sci 2004;59A:255-263.

4. Bergman H, Ferrucci L, Guralnik J et al. Frailty: An emerging research and clinical paradigm-issues and controversies. J Gerontol A Biol Sci Med Sci 2007;62A:731-737.

5. Ensrud KE, Ewing SK, Taylor BC et al. Frailty and risk of falls, fracture, and mortality in older women: The Study of Osteoporotic Fractures. J Gerontol A Biol Sci Med Sci 2007;62A:744-751.

6. Woods NF, LaCroix AZ, Gray SL et al. Frailty: Emergence and consequences in women aged 65 and older in the Women's Health Initiative Observational Study. J Am Geriatr Soc 2005;53:1321-1330.

7. Bandeen-Roche K, Xue QL, Ferrucci L et al. Phenotype of frailty: Characterization in the Women's Health and Aging Studies. J Gerontol A Biol Sci Med Sci 2006;61A:262-266.

8. Cawthon PM, Marshall LM, Michael Y et al. Frailty in older men: Prevalence, progression, and relationship with mortality. J Am Geriatr Soc 2007;55:1216-1223.

9. Cacciatore F, Abete P, Mazzella F et al. Frailty predicts long-term mortality in elderly subjects with chronic heart failure. Eur J Clin Invest 2005;35:723 -730 .

10. Gill TM, Gahbauer EA, Allore HG et al. Transitions between frailty states among community-living older persons. Arch Intern Med 2006;166:418423.

11. Afilalo J, Karunananthan S, Eisenberg MJ et al. Role of frailty in patients with cardiovascular disease. Am J Cardiol 2009;103:1616-1621.

12. Newman AB, Gottdiener JS, McBurnie MA et al. Associations of subclinical cardiovascular disease with frailty. J Gerontol A Biol Sci Med Sci 2001;56A:M158-M166.

13. Morley JE. Diabetes, sarcopenia, and frailty. Clin Geriatr Med 2008;24:455-469.

14. Blaum CS, Xue QL, Tian J et al. Is hyperglycemia associated with frailty status in older women? J Am Geriatr Soc 2009;57:840-847.

15. Chen CY, Wu SC, Chen LJ et al. The prevalence of subjective frailty and factors associated with frailty in Taiwan. Arch Gerontol Geriatr 2010;50 (Suppl 1):S43-S47. 
16. Santos-Eggimann B, Karmaniola A, Seematter-Bagnoud L et al. The Lausanne cohort Lc65+: A population-based prospective study of the manifestations, determinants and outcomes of frailty. BMC Geriatr 2008;8:20.

17. Wietlisbach V, Paccaud F, Rickenbach $\mathrm{M}$ et al. Trends in cardiovascular risk factors (1984-1993) in a Swiss region: Results of three population surveys. Prev Med 1997;26:523-533.

18. Santos-Eggimann B, Cuenoud P, Spagnoli J et al. Prevalence of frailty in middle-aged and older community-dwelling Europeans living in 10 countries. J Gerontol A Biol Sci Med Sci 2009;64A:675-681.

19. Crimmins EM, Kim JK, Sole-Auro A. Gender differences in health: Results from SHARE, ELSA and HRS. Eur J Public Health 2011;21:81-91.

20. Avendano M, Glymour MM, Banks J et al. Health disadvantage in US adults aged 50 to 74 years: A comparison of the health of rich and poor Americans with that of Europeans. Am J Public Health 2009;99:540-548.

21. Semba RD, Blaum CS, Bartali B et al. Denture use, malnutrition, frailty, and mortality among older women living in the community. J Nutr Health Aging 2006;10:161-167.

22. Masel MC, Graham JE, Reistetter TA et al. Frailty and health related quality of life in older Mexican Americans. Health Qual Life Outcomes 2009;7:70.

23. Ensrud KE, Ewing SK, Cawthon PM et al. A comparison of frailty indexes for the prediction of falls, disability, fractures, and mortality in older men. J Am Geriatr Soc 2009;57:492-498.

24. Kiely DK, Cupples LA, Lipsitz LA. Validation and comparison of two frailty indexes: The MOBILIZE Boston Study. J Am Geriatr Soc 2009;57:1532-1539.

25. Syddall H, Roberts HC, Evandrou M et al. Prevalence and correlates of frailty among community-dwelling older men and women: Findings from the Hertfordshire Cohort Study. Age Ageing 2010;39:197-203.

26. Drey M, Pfeifer K, Sieber CC et al. The Fried frailty criteria as inclusion criteria for a randomized controlled trial: Personal experience and literature review. Gerontology 2011;57:11-18.

27. Xue QL, Bandeen-Roche K, Varadhan R et al. Initial manifestations of frailty criteria and the development of frailty phenotype in the Women's Health and Aging Study II. J Gerontol A Biol Sci Med Sci 2008;63A:984-990.

28. Rantanen T, Guralnik JM, Foley D et al. Midlife hand grip strength as a predictor of old age disability. JAMA 1999;281:558-560.

29. Rantanen T, Volpato S, Ferrucci L et al. Handgrip strength and cause-specific and total mortality in older disabled women: Exploring the mechanism. J Am Geriatr Soc 2003;51:636-641.

30. Weiss CO. Frailty and chronic diseases in older adults. Clin Geriatr Med 2011;27:39-52.

31. Cetinus E, Buyukbese MA, Uzel M et al. Hand grip strength in patients with type 2 diabetes mellitus. Diabetes Res Clin Pract 2005;70:278-286.

32. Whooley MA, Browner WS. Association between depressive symptoms and mortality in older women Study of Osteoporotic Fractures Research Group. Arch Intern Med 1998;158:2129-2135.
33. Tell GS, Fried LP, Hermanson B et al. Recruitment of adults 65 years and older as participants in the Cardiovascular Health Study. Ann Epidemiol 1993;3:358-366.

34. Jacobsen SJ, Mahoney DW, Redfield MM et al. Participation bias in a population-based echocardiography study. Ann Epidemiol 2004;14:579_ 584.

35. Galea S, Tracy M. Participation rates in epidemiologic studies. Ann Epidemiol 2007;17:643-653.

36. Bisgard KM, Folsom AR, Hong CP et al. Mortality and cancer rates in nonrespondents to a prospective study of older women: 5-year follow-up. Am J Epidemiol 1994;139:990-1000.

37. Kauppi M, Sokka T, Hannonen P. Survey nonresponse is associated with increased mortality in patients with rheumatoid arthritis and in a community population. J Rheumatol 2005;32:807-810.

38. Ekholm O, Gundgaard J, Rasmussen NK et al. The effect of health, socioeconomic position, and mode of data collection on non-response in health interview surveys. Scand J Public Health 2010;38:699-706.

39. Kriegsman DM, Penninx BW, van Eijk JT et al. Self-reports and general practitioner information on the presence of chronic diseases in community dwelling elderly A study on the accuracy of patients' self-reports and on determinants of inaccuracy. J Clin Epidemiol. 1996;49:1407-1417.

40. Psaty BM, Kuller LH, Bild D et al. Methods of assessing prevalent cardiovascular disease in the Cardiovascular Health Study. Ann Epidemiol 1995;5:270-277.

41. Bergmann MM, Byers T, Freedman DS et al. Validity of self-reported diagnoses leading to hospitalization: A comparison of self-reports with hospital records in a prospective study of American adults. Am J Epidemiol 1998;147:969-977.

42. Yamagishi K, Ikeda A, Iso $\mathrm{H}$ et al. Self-reported stroke and myocardial infarction had adequate sensitivity in a population-based prospective study JPHC (Japan Public Health Center)-based Prospective Study. J Clin Epidemiol 2009;62:667-673.

43. Okura Y, Urban LH, Mahoney DW et al. Agreement between self-report questionnaires and medical record data was substantial for diabetes, hypertension, myocardial infarction and stroke but not for heart failure. J Clin Epidemiol 2004;57:1096-1103.

44. Leikauf J, Federman AD. Comparisons of self-reported and chart-identified chronic diseases in inner-city seniors. J Am Geriatr Soc 2009;57:12191225 .

45. Goldman N, Lin IF, Weinstein M et al. Evaluating the quality of selfreports of hypertension and diabetes. J Clin Epidemiol 2003;56:148-154.

46. Mathiowetz V, Kashman N, Volland G et al. Grip and pinch strength: Normative data for adults. Arch Phys Med Rehabil 1985;66:69-74.

47. Sequeira MM, Rickenbach M, Wietlisbach V et al. Physical activity assessment using a pedometer and its comparison with a questionnaire in a large population survey. Am J Epidemiol 1995;142:989-999. 